\title{
GLP-1-Analagon senkt Gewicht unabhängig vom Ausgangs-BMI
}

\author{
Ein GLP-1-Analogon unterstützt \\ BMI-unabhängig beim Abneh- \\ men und kann Typ-2-Diabetes \\ aufhalten. Das ist das \\ Ergebnis einer Post-hoc-Ana- \\ lyse der Studie SCALE. Aber \\ es gibt auch unerwünschte \\ Nebeneffekte.
}

Immer mehr Menschen in Industrieländern leiden an Übergewicht oder Adipositas. Lebensstiländerungen in Form einer Ernährungsumstellung und vermehrten körperlichen Aktivität erzielen oft nicht den gewünschten langfristigen $\mathrm{Ge}$ wichtsverlust. Hoffnung besteht deshalb, dieses Problems auch mit Medikamenten Herr werden zu können. Das Antidiabetikum Liraglutid ist inzwischen auch in höherer Dosierung als in der Diabetestherapie $(3 \mathrm{mg} / \mathrm{d})$ zur Gewichtsreduktion bei Nichtdiabetikern zugelassen (Saxen$\left.\mathrm{da}^{\star}\right)$, und zwar bei Personen mit einem $\mathrm{BMI} \geq 30 \mathrm{~kg} / \mathrm{m}^{2}$ oder einem BMI zwischen 27 und $30 \mathrm{~kg} / \mathrm{m}^{2}$, falls zusätzlich mindestens eine gewichtsbedingte Begleiterkrankung vorliegt. Als Analogon des "Glucagon-like peptide 1“ (GLP-1) steigert die Substanz glukoseabhängig die Insulinsekretion und führt über eine Verzögerung der Magenentleerung und Verringerung des Appetits zur Gewichtsreduktion. Ob Liraglutid das Körpergewicht auch unabhängig vom Ausgangsgewicht gleichmäßig zu senken vermag, haben Wissenschaftler um Prof. Sten Madsbad von der Universität Kopenhagen nun in einer Post-hocAnalyse der vom Hersteller des Präparats Novo Nordisk finanzierten randomisierten und doppelblinden SCALE-Studie (Satiety and Clinical Adioposity - Liraglutide Evidence) untersucht. Die Ergebnisse stellte der Studienleiter beim EASD-Kongress in München vor.
Ziel der Analyse sei gewesen, die Sicherheit und Effektivität des Medikaments in vier BMI-Kategorien $\left(27-29,9 \mathrm{~kg} / \mathrm{m}^{2}, 30-34,9\right.$ $\mathrm{kg} / \mathrm{m}^{2}, 35-39,9 \mathrm{~kg} / \mathrm{m}^{2}$ sowie $\left.\geq 40 \mathrm{~kg} / \mathrm{m}^{2}\right)$ zu evaluieren, berichtete er. Die 2.254 Teilnehmer hatten zu Studienbeginn Prädiabetes, diagnostiziert anhand einer gestörten Nüchternglukose (100-125 $\mathrm{mg} / \mathrm{dl}$ ) und/oder einer gestörten Glukosetoleranz (140-199 mg/ dl) und/oder eines $\mathrm{HbA}_{1 \mathrm{c}}$ zwischen 5,7 und 6,4\%. 1.505 Teilnehmer erhielten $3 \mathrm{mg} / \mathrm{d}$ Liraglutid, 749 Placebo, jeweils ergänzt durch eine Lebensstilintervention mit kalorienreduzierten Ernährung und vermehrter körperlicher Aktivität.

\section{Gewichtsverlust in allen BMI-Subgruppen}

Während der dreijähriger Behandlung verloren die Teilnehmer in der Liraglutid-Gruppe im Schnitt 6,17\% an Gewicht, mit Placebo $1,84 \%$. Die Veränderungen im BMI seien in den einzelnen Kategorien nicht signifikant unterschiedlich ausgefallen, berichtete Madsbad. Auch erreichten unabhängig von der Kategorie signifikant mehr Patienten einen Normblutzuckerwert als mit Placebo (mit steigendem BMI: $67 \%, 67 \%, 70 \%$ und $63 \%$ vs. $36 \%$, $34 \%, 40 \%$ und $33 \%$ ). Ebenfalls konsistent in allen BMI-Klassen verbesserte sich die Insulinsensitivität der Teilnehmer mit Liraglutid. Im Studiezeitraum entwickelten 26 Patienten der Liraglutid- und 46 Teilnehmer der Placebo-Gruppe Typ-2-Diabetes. Die geschätzte Zeit bis zur Manifestation eines Diabetes wurde mit dem GLP-1-Analogon um im Mittel das 2,7-Fache herausgezögert, was einer Hazard Ratio von 0,2 entspricht.

\section{Gastrointestinale Beschwerden}

Ebenfalls keinen Einfluss scheint Madsbad zufolge das Ausgangsgewicht auf die Häufigkeit von Nebenwirkungen zu haben. Generell sei Liraglutid in allen BMI-Subgruppen gut vertragen worden. Allerdings kam es unter der Therapie vermehrt zu gastrointestinalen Beschwerden. Nicht überraschend seien darunter auch Hypoglykämien häufiger aufgetreten als in der Placebogruppe. Es handele sich bei Liraglutid immerhin um eine der am stärksten auf die Insulinsekretion wirkenden Substanzen, so Madsbad. So kam es bei $17 \%$ der Patienten mit einem BMI $\geq 40$ mindestens einmal zu einer Hypoglykämie, mit Placebo bei 5,7\%. In der niedrigsten BMI-Kategorie sind die Zahlen entsprechend $17,9 \%$ vs. $4,3 \%$. Basierend auf diesen $\mathrm{Da}-$ ten gebe es keine Anhaltspunkte, dass Liraglutid in einzelnen Gewichtskategorien unterschiedlich auf die Glukosekontrolle und Betazellfunktion wirke, schloss Madsbad. Allerdings, so betonte er abschließend, habe diese Post-hoc-Analyse weder die Power, noch sei die Studie primär dafür angelegt worden, die Effektivität der Substanz bzgl. der Blutzuckerkontrolle über unterschiedliche BMI-Subgruppen hinweg zu vergleichen.

Veronika Schlimpert

Quelle: Symposium „Pills or surgery for weight loss?" Results from the SCALE obesity and prediabetes trial, 14. 9.2016 EASD-Kongress in München 